LBNL-40770

UCB-PTH-97/29

hep-th/9705226

May 1997

\title{
Self-Duality in Nonlinear Electromagnetism 冈1
}

\author{
Mary K. Gaillard and Bruno Zumino \\ Physics Department, University of California, and \\ Theoretical Physics Group, Lawrence Berkeley National Laboratory, \\ Berkeley, California 94720
}

\begin{abstract}
We discuss duality invariant interactions between electromagnetic fields and matter. The case of scalar fields is treated in some detail.
\end{abstract}

\footnotetext{
${ }^{*}$ Contribution to the memorial volume for D.V. Volkov.

†This work was supported in part by the Director, Office of Energy Research, Office of High Energy and Nuclear Physics, Division of High Energy Physics of the U.S. Department of Energy under Contract DE-AC03-76SF00098 and in part by the National Science Foundation under grant PHY-95-14797.
} 


\section{Disclaimer}

This document was prepared as an account of work sponsored by the United States Government. While this document is believed to contain correct information, neither the United States Government nor any agency thereof, nor The Regents of the University of California, nor any of their employees, makes any warranty, express or implied, or assumes any legal liability or responsibility for the accuracy, completeness, or usefulness of any information, apparatus, product, or process disclosed, or represents that its use would not infringe privately owned rights. Reference herein to any specific commercial products process, or service by its trade name, trademark, manufacturer, or otherwise, does not necessarily constitute or imply its endorsement, recommendation, or favoring by the United States Government or any agency thereof, or The Regents of the University of California. The views and opinions of authors expressed herein do not necessarily state or reflect those of the United States Government or any agency thereof, or The Regents of the University of California.

Lawrence Berkeley Laboratory is an equal opportunity employer. 


\section{Duality rotations in four dimensions}

The invariance of Maxwell's equations under "duality rotations" has been known for a long time. In relativistic notation these are rotations of the electromagnetic field strength $F_{\mu \nu}$ into its dual, which is defined by

$$
\tilde{F}_{\mu \nu}=\frac{1}{2} \epsilon_{\mu \nu \lambda \sigma} F^{\lambda \sigma}, \quad \tilde{\tilde{F}}_{\mu \nu}=-F_{\mu \nu} .
$$

This invariance can be extended to electromagnetic fields in interaction with the gravitational field, which does not transform under duality. It is present in ungauged extended supergravity theories, in which case it generalizes to a nonabelian group [1]. In [2, 3] we studied the most general situation in which duality invariance of this type can occur. More recently [4] the duality invariance of the Born-Infeld theory, suitably coupled to the dilaton and axion [5], has been studied in considerable detail. In the present note we will show that most of the results of [4, [0] follow quite easily from our earlier general discussion. We shall also present some new results that were not made explicit in [2, 20, especially some properties of the scalar fields.

We begin by recalling and completing some basic results of our paper [2, 3]. Consider a Lagrangian which is a function of $n$ real field strengths $F_{\mu \nu}^{a}$ and of some other fields $\chi^{i}$ and their derivatives $\chi_{\mu}^{i}=\partial_{\mu} \chi^{i}$ :

$$
L=L\left(F^{a}, \chi^{i}, \chi_{\mu}^{i}\right) .
$$

Since

$$
F_{\mu \nu}^{a}=\partial_{\mu} A_{\nu}^{a}-\partial_{\nu} A_{\mu}^{a},
$$

we have the Bianchi identities

$$
\partial^{\mu} \tilde{F}_{\mu \nu}^{a}=0 .
$$

On the other hand, if we define

$$
\tilde{G}_{\mu \nu}^{a}=\frac{1}{2} \epsilon_{\mu \nu \lambda \sigma} G^{a \lambda \sigma} \equiv 2 \frac{\partial L}{\partial F_{a}^{\mu \nu}},
$$

we have the equations of motion

$$
\partial^{\mu} \tilde{G}_{\mu \nu}^{a}=0 .
$$


We consider an infinitesimal transformation of the form

$$
\begin{aligned}
\delta\left(\begin{array}{l}
F \\
G
\end{array}\right) & =\left(\begin{array}{ll}
A & B \\
C & D
\end{array}\right)\left(\begin{array}{l}
F \\
G
\end{array}\right), \\
\delta \chi^{i} & =\xi^{i}(\chi),
\end{aligned}
$$

where $A, B, C, D$ are real $n \times n$ constant infinitesimal matrices and $\xi^{i}(\chi)$ functions of the fields $\chi^{i}$ (but not of their derivatives), and ask under what circumstances the system of the equations of motion (1.4) and (1.6), as well as the equation of motion for the fields $\chi^{i}$ are invariant. The analysis of [2] shows that this is true if the matrices satisfy

$$
A^{T}=-D, \quad B^{T}=B, \quad C^{T}=C,
$$

(where the superscript $T$ denotes the transposed matrix) and in addition the Lagrangian changes under (1.7) and (1.8) as

$$
\delta L=\frac{1}{4}(F C \tilde{F}+G B \tilde{G}) .
$$

The relations (1.9) show that (1.7) is an infinitesimal transformation of the real noncompact symplectic group $S p(2 n, R)$ which has $U(n)$ as maximal compact subgroup. The finite form is

$$
\left(\begin{array}{l}
F^{\prime} \\
G^{\prime}
\end{array}\right)=\left(\begin{array}{ll}
a & b \\
c & d
\end{array}\right)\left(\begin{array}{l}
F \\
G
\end{array}\right),
$$

where the $n \times n$ real submatrices satisfy

$$
c^{T} a=a^{T} c, \quad b^{T} d=d^{T} b, \quad d^{T} a-b^{T} c=1 .
$$

Notice that the Lagrangian is not invariant. In [2] we showed, however, that the derivative of the Lagrangian with respect to an invariant parameter is invariant. The invariant parameter could be a coupling constant or an external background field, such as the gravitational field, which does not change under duality rotations. It follows that the energy-momentum tensor, which can be obtained as the variational derivative of the Lagrangian with respect to the gravitational field, is invariant under duality rotations. No explicit check of its invariance, as was done in [ []]-[7], is necessary.

The symplectic transformation (1.11) can be written in a complex basis as

$$
\left(\begin{array}{c}
F^{\prime}+i G^{\prime} \\
F^{\prime}-i G^{\prime}
\end{array}\right)=\left(\begin{array}{ll}
\phi_{0} & \phi_{1}^{*} \\
\phi_{1} & \phi_{0}^{*}
\end{array}\right)\left(\begin{array}{c}
F+i G \\
F-i G
\end{array}\right),
$$


where $*$ means complex conjugation and the submatrices satisfy

$$
\phi_{0}^{T} \phi_{1}=\phi_{1}^{T} \phi_{0}, \quad \phi_{0}^{\dagger} \phi_{0}-\phi_{1}^{\dagger} \phi_{1}=1 .
$$

The relation between the real and the complex basis is

$$
\begin{array}{rlrl}
2 a & =\phi_{0}+\phi_{0}^{*}+\phi_{1}+\phi_{1}^{*}, & -2 i b & =\phi_{0}-\phi_{0}^{*}+\phi_{1}-\phi_{1}^{*}, \\
2 i c & =\phi_{0}-\phi_{0}^{*}-\phi_{1}+\phi_{1}^{*}, \quad 2 d & =\phi_{0}+\phi_{0}^{*}-\phi_{1}-\phi_{1}^{*} .
\end{array}
$$

In [2, [3] we also described scalar fields valued in the quotient space $S p(2 n, R) / U(n)$. The quotient space can be parameterized by a complex symmetric $n \times n$ matrix $K=K^{T}$ whose real part has positive eigenvalues, or equivalently by a complex symmetric matrix $Z=Z^{T}$ such that $Z^{\dagger} Z$ has eigenvalues smaller than 1 . They are related by

$$
K=\frac{1-Z^{*}}{1+Z^{*}}, \quad Z=\frac{1-K^{*}}{1+K^{*}} .
$$

These formulae are the generalization of the well-known map between the Lobachevskiı̌ unit disk and the Poincaré upper half-plane: $Z$ corresponds to the single complex variable parameterizing the unit disk; $i K$ to the one parameterizing the upper half plane.

$$
\begin{aligned}
& \text { Under } \operatorname{Sp}(2 n, R) \\
& K \rightarrow K^{\prime}=(-i c+d K)(a+i b K)^{-1}, \quad Z \rightarrow Z^{\prime}=\left(\phi_{1}+\phi_{0}^{*} Z\right)\left(\phi_{0}+\phi_{1}^{*} Z\right)^{-1},
\end{aligned}
$$

or, infinitesimally,

$$
\delta K=-i C+D K-K A-i K B K, \quad \delta Z=V+T^{*} Z-Z T-i Z V^{*} Z,
$$

where

$$
T=-T^{\dagger}, \quad V=V^{T} .
$$

The invariant nonlinear kinetic term for the scalar fields can be obtained from the Kähler metric [8]

$$
\operatorname{Tr}\left(d K^{*} \frac{1}{K+K^{*}} d K \frac{1}{K+K^{*}}\right)=\operatorname{Tr}\left(d Z \frac{1}{1-Z^{*} Z} d Z^{*} \frac{1}{1-Z Z^{*}}\right)
$$

which follows from the Kähler potential

$$
\operatorname{Tr} \ln \left(1-Z Z^{*}\right) \quad \text { or } \quad \operatorname{Tr} \ln \left(K+K^{*}\right),
$$

which are equivalent up to a Kähler transformation. It is not hard to show that the metric (1.20) is positive definite. Throughout this paper we assume a flat background space-time metric; the generalization to a nonvanishing gravitational field is straightforward [2]-[5]. 


\section{Born-Infeld theory}

As a particularly simple example we consider the case when there is only one tensor $F_{\mu \nu}$ and no additional fields. Our equations become

$$
\begin{gathered}
\tilde{G}=2 \frac{\partial L}{\partial F}, \\
\delta F=\lambda G, \quad \delta G=-\lambda F
\end{gathered}
$$

and

$$
\delta L=\frac{1}{4} \lambda(G \tilde{G}-F \tilde{F}) .
$$

We have restricted the duality transformation to the compact subgroup $U(1) \cong$ $S O(2)$, as appropriate when no additional fields are present. So $A=D=$ $0, B=-C=\lambda$.

Since $L$ is a function of $F$ alone, we can also write

$$
\delta L=\delta F \frac{\partial L}{\partial F}=\lambda G \frac{1}{2} \tilde{G} .
$$

Comparing (2.3) and (2.4), which must agree, we find

$$
G \tilde{G}+F \tilde{F}=0 .
$$

Together with (2.1), this is a partial differential equation for $L(F)$, which is the condition for the theory to be duality invariant. If we introduce the complex field

$$
M=F-i G,
$$

(2.5) can also be written as

$$
M \widetilde{M}^{*}=0 .
$$

Clearly, Maxwell's theory in vacuum satisfies (2.5), or (2.7), as expected. A more interesting example is the Born-Infeld theory [6], given by the Lagrangian

$$
L=\frac{1}{g^{2}}\left(-\Delta^{\frac{1}{2}}+1\right)
$$

where

$$
\Delta=-\operatorname{det}\left(\eta_{\mu \nu}+g F_{\mu \nu}\right)=1+\frac{1}{2} g^{2} F^{2}-g^{4}\left(\frac{1}{4} F \tilde{F}\right)^{2} .
$$

For small values of the coupling constant $g$ (or for weak fields) $L$ approaches the Maxwell Lagrangian. We shall use the abbreviation

$$
\beta=\frac{1}{4} F \tilde{F} .
$$


Then

$$
\begin{gathered}
\frac{\partial \Delta}{\partial F}=g^{2} F-\beta g^{4} \tilde{F}, \\
\tilde{G}=2 \frac{\partial L}{\partial F}=-\Delta^{-\frac{1}{2}}\left(F-\beta g^{2} \tilde{F}\right),
\end{gathered}
$$

and

$$
G=\Delta^{-\frac{1}{2}}\left(\tilde{F}+\beta g^{2} F\right) .
$$

Using (2.12) and (2.13), it is very easy to check that $G \tilde{G}=-F \tilde{F}$ : the BornInfeld theory is duality invariant. It is also not too difficult to check that $\partial L / \partial g^{2}$ is actually invariant under (2.2) and the same applies to $L-\frac{1}{4} F \tilde{G}$ (which in this case turns out to be equal to $-g^{2} \partial L / \partial g^{2}$ ). These invariances are expected from our general theory.

It is natural to ask oneself whether the Born-Infeld theory is the most general physically acceptable solution of (2.5). This was investigated in [4] where a negative result was reached: more general Lagrangians satisfy (2.5), the arbitrariness depending on a function of one variable.

\section{Schrödinger's formulation of Born's theory}

Schrödinger [7] noticed that, for the Born-Infeld theory (2.8), $F$ and $G$ satisfy not only (2.5) [or (2.7)], but also the more restrictive relation

$$
M(M \widetilde{M})-\widetilde{M} M^{2}=\frac{g^{2}}{8} \widetilde{M}^{*}(M \widetilde{M})^{2} .
$$

We have verified this by an explicit, although lengthy, calculation using (2.6), (2.12), (2.13) and (2.9). Schrödinger did not give the details of the calculation, presenting instead convincing arguments based on particular choices of reference systems. One can write (3.1) as

$$
\frac{\partial \mathcal{L}}{\partial M}=g^{2} \widetilde{M}^{*},
$$

where

$$
\mathcal{L}=4 \frac{M^{2}}{(M \widetilde{M})},
$$

and Schrödinger proposed $\mathcal{L}$ as the Lagrangian of the theory, instead of (2.8). Of course, $\mathcal{L}$ is a Lagrangian in a different sense than $L$, which is a field Lagrangian in the usual sense. Multiplying (3.1) by $M$ and saturating the 
unwritten indices $\mu \nu$, the left hand side vanishes, so that (2.7) follows. Using (3.1) it is easy to see that $\mathcal{L}$ is pure imaginary: $\mathcal{L}=-\mathcal{L}^{*}$. Schrödinger also pointed out that, if we introduce a map

$$
\frac{1}{g^{2}} \frac{\partial \mathcal{L}}{\partial M}=f(M),
$$

so that (3.1) or (3.2) can be written as

$$
f(M)=\widetilde{M}^{*},
$$

the square of the map is the identity map

$$
f(f(M))=M .
$$

This, together with the properties

$$
f(\widetilde{M})=-\tilde{f}(M), \quad f\left(M^{*}\right)=f(M)^{*},
$$

ensures the consistency of (3.1). Schrödinger used the Lagrangian (3.3) to construct a conserved, symmetric energy-momentum tensor. We have checked that, when suitably normalized, his energy-momentum tensor agrees with that of Born and Infeld up to an additive term proportional to $\eta_{\mu \nu}$.

Schrödinger's formulation is very clever and elegant and it has the advantage of being manifestly covariant under the duality rotation $M \rightarrow M e^{i \lambda}$ which is the finite form of (2.2). It is also likely that, as he seems to imply, his formulation is fully equivalent to the Born-Infeld theory (2.8), which would mean that the more restrictive equation (3.1) eliminates the remaining ambiguity in the solutions of (2.7). This virtue could actually be a weakness if one is looking for more general duality invariant theories.

\section{Axion, dilaton and $S L(2, R)$}

It is well known that, if there are additional scalar fields which transform nonlinearly, the compact group duality invariance can be enhanced to a duality invariance under a larger noncompact group (see, e.g., [2] and references therein). In the case of the Born-Infeld theory, just as for Maxwell's theory, one complex scalar field suffices to enhance the $U(1) \cong S O(2)$ invariance to the $S U(1,1) \cong S L(2, R)$ noncompact duality invariance. This is pointed out 
in [5], but it also follows the considerations of our paper [2]. We shall use the letter $S$ instead of $K$ for the scalar field, which, in the example under consideration, is a single complex field, not an $n \times n$ matrix. In today's more standard notation

$$
S=S_{1}-i S_{2}=e^{-\phi}-i a, \quad S_{1}>0,
$$

where $\phi$ is the dilaton and $a$ is the axion. For $S L(2, R) \cong S p(2, R)$, the matrices $A, B, C, D$ are real numbers and $A=-D, B$ and $C$ are independent. Then the infinitesimal $S L(2, R)$ transformation is

$$
\delta S=-2 A S-i B S^{2}-i C .
$$

For the $S O(2) \cong U(1)$ subgroup, $A=0, B=-C=\lambda$,

$$
\delta S=-i \lambda S^{2}+i \lambda .
$$

The scalar kinetic term, proportional to

$$
\frac{\partial_{\mu} S^{*} \partial^{\mu} S}{\left(S+S^{*}\right)^{2}}
$$

is invariant under the nonlinear transformation (4.2) which, in terms of $S_{1}, S_{2}$, takes the form

$$
\delta S_{1}=-2 A S_{1}-i B S_{1} S_{2}, \quad \delta S_{2}=-2 A S_{2}+B\left(S_{1}^{2}-S_{2}^{2}\right)+C .
$$

The full noncompact duality transformation on $F_{\mu \nu}$ is now

$$
\delta F=A F+B G, \quad \delta G=D F+D G, \quad D=-A,
$$

and we are seeking a Lagrangian $\hat{L}(F, S)$ which satisfies

$$
\delta \hat{L}=\frac{1}{4}(F C \tilde{F}+G B \tilde{G}),
$$

where

$$
\delta \hat{L}=\delta F \frac{\partial \hat{L}}{\partial F}+\delta S_{1} \frac{\partial \hat{L}}{\partial S_{1}}+\delta S_{2} \frac{\partial \hat{L}}{\partial S_{2}}
$$

and now

$$
\tilde{G}=2 \frac{\partial \hat{L}}{\partial F}
$$


Equating (4.7) and (4.8) we see that $\hat{L}$ must satisfy

$$
\frac{1}{4}(B G \tilde{G}-C F \tilde{F})+\frac{1}{2} A F \tilde{G}+\delta S_{1} \frac{\partial \hat{L}}{\partial S_{1}}+\delta S_{2} \frac{\partial \hat{L}}{\partial S_{2}}=0
$$

This equation can be solved as follows. Assume that $L(\mathcal{F})$ satisfies (2.1) and (2.5), i.e.

$$
\mathcal{G} \tilde{\mathcal{G}}+\mathcal{F} \tilde{\mathcal{F}}=0
$$

where

$$
\tilde{\mathcal{G}}=2 \frac{\partial \mathcal{L}}{\partial \mathcal{F}}
$$

For instance, the Born-Infeld Lagrangian $L(\mathcal{F})$ does this. Then

$$
\hat{L}(S, F)=L\left(S_{1}^{\frac{1}{2}} F\right)+\frac{1}{4} S_{2} F \tilde{F}
$$

satisfies (4.10). Indeed

$$
\frac{\partial \hat{L}(S, F)}{\partial F}=\frac{\partial L}{\partial \mathcal{F}} S_{1}^{\frac{1}{2}}+\frac{1}{2} S_{2} \tilde{F}
$$

So

$$
\begin{aligned}
\tilde{G} & =\tilde{\mathcal{G}} S_{1}^{\frac{1}{2}}+S_{2} \tilde{F}, \\
G & =\mathcal{G} S_{1}^{\frac{1}{2}}+S_{2} F
\end{aligned}
$$

where we have defined

$$
\mathcal{F}=S_{1}^{\frac{1}{2}} F
$$

and $\tilde{\mathcal{G}}$ is given by (4.12). Now

$$
G \tilde{G}=\mathcal{G} \tilde{\mathcal{G}} S_{1}+S_{2}^{2} F \tilde{F}+2 S_{2} \mathcal{F} \tilde{\mathcal{G}} .
$$

Using (4.11) in this equation we find

$$
G \tilde{G}=\left(S_{2}^{2}-S_{1}^{2}\right) F \tilde{F}+2 S_{2} \mathcal{F} \tilde{\mathcal{G}} .
$$

We also have

$$
F \tilde{G}=\mathcal{F} \tilde{\mathcal{G}}+S_{2} F \tilde{F} .
$$

On the other hand, since

$$
\frac{\partial L}{\partial S_{1}^{\frac{1}{2}}}=\frac{\partial \mathcal{L}}{\partial \mathcal{F}} F=\frac{1}{2} \tilde{\mathcal{G}} F
$$


we obtain

$$
\frac{\partial \hat{L}}{\partial S_{1}}=\frac{\partial L}{\partial S_{1}^{\frac{1}{2}}} \frac{1}{2} S_{1}^{-\frac{1}{2}}=\frac{1}{4} \tilde{\mathcal{G}} S_{1}^{-\frac{1}{2}} F=\frac{1}{4} \tilde{\mathcal{G}} \mathcal{F} S_{1}^{-1}
$$

In addition

$$
\frac{\partial \hat{L}}{\partial S_{2}}=\frac{1}{4} F \tilde{F}
$$

Using (4.19), (4.20), (4.22) and (4.23), together with (4.5), we see that (4.10) is satisfied. It is easy to check that the scale invariant combinations $\mathcal{F}$ and $\mathcal{G}$, given by (4.17) and (4.12) have the very simple transformation law

$$
\delta \mathcal{F}=S_{1} B \mathcal{G}, \quad \delta \mathcal{G}=-S_{1} B \mathcal{F}
$$

i.e., they transform according to the $U(1) \cong S O(2)$ compact subgroup just as $F$ and $G$ in (2.2), but with the parameter $\lambda$ replaced by $S_{1} B$. If $L(\mathcal{F})$ is the Born-Infeld Lagrangian, the theory with scalar fields given by $\hat{L}$ in (4.13) can also be reformulated à la Schrödinger. From (4.16) and (4.17) solve for $\mathcal{F}$ and $\mathcal{G}$ in terms of $F, G, S_{1}$ and $S_{2}$. Then $\mathcal{M}=\mathcal{F}-i \mathcal{G}$ must satisfy the same equation (3.1) that $M$ does when no scalar fields are present.

\section{Connections to string theory}

The duality rotations considered here are relevant to effective field theories from superstrings. The supersymmetric extension [9] of the Lagrangian (4.13) with $L(\mathcal{F})=-\frac{1}{4} \mathcal{F}^{2}$ describes the dilaton plus Yang-Mills sector of effective $N=1$ supergravity theories obtained from superstrings in the weak coupling $\left(S_{1} \rightarrow \infty\right)$ limit. The $S L(2, Z)$ subgroup of $S L(2, R)$ that is generated by the elements $4 \pi S \rightarrow 1 / 4 \pi S$ and $S \rightarrow S-i / 4 \pi$ relates different string theories 10 to one another. The generalization of [2] to two dimensional theories [11] has been used to derive the Kähler potential for moduli and matter fields in

effective field theories from superstrings. In this case the scalars are valued on a coset space $\mathcal{K} / \mathcal{H}, \mathcal{K} \in S O(n, n), \mathcal{H} \in S O(n) \times S O(n)$. The kinetic energy is invariant under $\mathcal{K}$, and the full classical theory is invariant under a subgroup of $\mathcal{K}$. String loop corrections reduces the invariance to a discrete subgroup that contains the $S L(2, Z)$ group generated by $T \rightarrow 1 / T, T \rightarrow T-i$, where $T$ is the squared radius of compactification in string units. 
Acknowledgements. We are grateful for the hospitality provided by the Isaac Newton Institute where this work was initiated. We thank Gary Gibbons, David Olive, Harold Steinacker, Kelly Stelle and Peter West for inspiring conversations. This work was supported in part by the Director, Office of Energy Research, Office of High Energy and Nuclear Physics, Division of High Energy Physics of the U.S. Department of Energy under Contract DEAC03-76SF00098 and in part by the National Science Foundation under grant PHY-95-14797.

\section{References}

[1] S. Ferrara, J. Scherk and B. Zumino, Nucl. Phys. B121: 393 (1977); E. Cremmer and B. Julia, Nucl. Phys. B159: 141 (1979).

[2] M.K. Gaillard and B. Zumino, Nucl. Phys. B193: 221 (1981).

[3] B. Zumino, Quantum Structure of Space and Time, Eds. M.J. Duff and C.J. Isham (Cambridge University Press) p. 363 (1982).

[4] G.W. Gibbons and D.A. Rasheed, Nucl. Phys. B454: 185 (1995).

[5] G.W. Gibbons and D.A. Rasheed, Phys. Lett. B365: 46 (1996).

[6] M. Born and L. Infeld, Proc. Roy. Soc. (London) A144: 425 (1934).

[7] E. Schrödinger, Proc. Roy. Soc. (London) A150: 465 (1935).

[8] P. Binétruy and M.K. Gaillard, Phys. Rev. D32: 931 (1985).

[9] P. Binétruy and M.K. Gaillard, Phys. Lett. B365: 87 (1996).

[10] J.H. Schwarz and A. Sen, Phys. Lett. B312: 105 (1993) and Nucl. Phys. B411: 35 (1994); M. Duff, Nucl. Phys. B442: 47 (1995); E. Witten, Nucl. Phys. B443: 85 (1995).

[11] S. Cecotti, S. Ferrara and L. Girardello, Nucl. Phys. B308: 436 (1988). 